\title{
« La politique et la poésie c'est trop pour un seul homme ». A propos de Glauber Rocha : cinéaste brésilien (1939-1981)
}

Angelina Peralva et Ismail Xavier

\section{OpenEdition}

Journals

Édition électronique

URL : http://journals.openedition.org/conflits/1896

DOI : $10.4000 /$ conflits. 1896

ISSN : $1777-5345$

Éditeur :

CCLS - Centre d'études sur les conflits lilberté et sécurité, L'Harmattan

Édition imprimée

Date de publication : 1 septembre 2005

Pagination : 185-203

ISBN : 2-7475-9476-9

ISSN : $1157-996 \mathrm{X}$

Référence électronique

Angelina Peralva et Ismail Xavier, « «La politique et la poésie c'est trop pour un seul homme ». A

propos de Glauber Rocha : cinéaste brésilien (1939-1981) 》, Cultures \& Conflits [En ligne], 59 | automne 2005, mis en ligne le 06 janvier 2010, consulté le 30 mars 2021. URL : http://journals.openedition.org/ conflits/1896; DOI : https://doi.org/10.4000/conflits.1896

Ce document a été généré automatiquement le 30 mars 2021.

Creative Commons License 


\title{
«La politique et la poésie c'est trop pour un seul homme ». A propos de Glauber Rocha : cinéaste brésilien (1939-1981)
}

\author{
Angelina Peralva et Ismail Xavier
}

1 Derrière l'idée de prendre appui sur Glauber Rocha pour poser à nouveau le problème de l'articulation entre culture et politique, il y a d'abord eu la volonté de retrouver une sorte de fil d'Ariane, présent dans ses films, permettant de suivre l'évolution des représentations sur la violence au Brésil, allant de la domination brute exercée par les grands propriétaires terriens à l'encontre des paysans dans la campagne aride du NordEst (Le Dieu noir et le diable blond, 1964ou Antonio das Mortes, 1969) à un vécu transversal à l'expérience urbaine (Terre en transe, 1967ou L'âge de la terre, 1980). Mais Rocha nous propose beaucoup plus. A travers plusieurs textes rendus publics dans des circonstances très diverses, dont l'un, «La transe de l'Amérique latine » est repris dans la version papier du présent dossier, et deux autres, «Esthétique de la faim " et "Esthétique du rêve " (deux manifestes majeurs dans son parcours), dans la version électronique, il mène une réflexion active (et tentative, car jamais entièrement aboutie) sur ce que pourrait être une esthétique capable de traduire la dynamique historique de l'Amérique latine de son époque.

2 Ayant fait le choix, pour des raisons pratiques, de traduire moi-même les textes présentés dans ce dossier, j'ai replongé, pour un court laps de temps, dans un exercice que j'ai souvent pratiqué par le passé, avant de l'abandonner pour la sociologie. J'ai toujours pensé à la traduction comme une infidélité - destinée à être, par les soins du traducteur, aussi limitée que possible. Infidélité, parce qu'on ne peut pas ne pas trahir le texte d'origine, dès lors que sa transposition dans la langue de traduction doit obéir aux règles d'intelligibilité propres à cette dernière; limitée, parce que l'enjeu est de trouver des compromis permettant de rapprocher les deux versions, en rendant compte non seulement d'un contenu mais aussi d'une forme, y compris lorsque cette 
forme parait maladroite ou inaboutie. Le traducteur, souvent, est tenté de faire mieux que l'auteur. J'ai évité de corriger Glauber autant que j'ai pu. Pourtant, son texte est parfois déroutant, ses expressions sont mal choisies, la syntaxe laisse à désirer. J'ai maintenu cette forme (pas tout à fait involontaire, me semble-t- il - mais comment en être sûre ?) en essayant de retrouver, en français, les écarts correspondants par rapport à ce qui en serait, en portugais, l'équivalent canonique. J'ai veillé à ce que cela ne nuise pas à la compréhension et à la fluidité de la lecture, mais j'ai évité d'aller au-delà. Tout compte fait, j'avoue avoir été séduite par cette écriture, en ce qu'elle oblige le lecteur à marquer des points d'arrêt, à se dire par moments qu'il n'a peut-être pas compris et à découvrir par là même les divers possibles de l'énoncé.

\section{« La transe de l'Amérique Latine » - entretien avec Glauber Rocha réalisé par Fernando Cardenas et René Capriles $^{1}$}

Glauber : Même si je fais un cinéma tourné vers la réalité sociale, je n'ai jamais admis la démagogie esthétique, sous quelque forme qui soit, face à un art politique ; car ce qui se passe, c'est qu'il y a des intellectuels, écrivains, artistes et cinéastes qui prennent prétexte de l'intention politique progressiste pour justifier une très mauvaise qualité de l'œuvre artistique. C'est une trahison que je n'admets pas, parce que je crois que le phénomène politique, le phénomène social n'acquièrent de l'importance artistique que lorsqu'ils sont exprimés à travers une œuvre d'art placée dans une perspective esthétique. C'est ce que dit la belle phrase de Brecht: «pour des idées nouvelles, des formes nouvelles ». Il n'y a pas d'autre issue. De telle sorte que je n'ai jamais aimé les théories d'art politique élaborées non seulement dans les termes du réalisme socialiste,mais aussi celles du réalisme critique tel que défini par Lukács et tous ceux qui ont écrit sur l'art révolutionnaire. Spécialement en Amérique latine, où il y a de grandes intentions dans les déclarations mais les résultats dénotent une aliénation complète vis-à-vis du processus cinématographique. C'est-à-dire que des auteurs qui combattent l'aliénation du point de vue sociopolitique font des films qui - pour la plupart d'entre eux - apparaissent profondément aliénés et qui sont, au fond, liés aux préjugés culturels colonialistes du cinéma américain ou européen. Quand j'ai fait Terra em transe, c'était surtout comme cinéaste, j'ai voulu que ce soit une rupture la plus radicale possible avec ce type d'influences, ce type d'aliénation cinématographique que l'on sent dans la critique et dans beaucoup de films. Bien sûr, il y a des exceptions, tous les critiques ou cinéastes n'agissent pas de cette manière, mais une bonne partie manifeste cette complaisance. Terra em transe a été la tentative d'obtenir dans le cinéma une expression complexe, indéfinie, mais propre et authentique à propos de tout ce qui pourrait être un cinéma d'Amérique latine.

On trouve déjà dans la littérature d'Amérique latine des exemples de ce type d'expérience: l'œuvre de Carpentier ou de García Marquez, celle de Neruda ou de Nicolás Guillén, mais pas dans le cinéma. J'ai fait Terra em transe avec cette intention. Non pas pour plaire au public ou à la critique; après dix ans d'expérience dans le cinéma, ayant financé moi-même mes films, je crois que le travail du cinéaste dans les pays sous-développés comprend aussi celui de producteur, diffuseur, publicitaire, tout. Faire du cinéma, pour moi, c'est un complexe total, je ne défends pas le mythe de l'artiste individuel, fermé, rien de tout ça. Je fais le film, je le produis, je le diffuse et je 
le projette. Alors, à ce niveau, il semblerait que le cinéaste ne peut plus vivre dans la dépendance. J'ai fait Terra em transe en aspirant à ce que ce fût une bombe. Lancée en toute intention. En m'attaquant aux préjugés d'une gauche académique, conservatrice, celle qui a manifesté à l'encontre du film une réaction névrosée, et cela a été positif. Au Brésil, le lancement du film a eu lieu au milieu d'une grande polémique, il a été interdit par la censure au motif qu'il s'agissait d'un film hautement subversif et immoral, l'accusation étant d'ordre politique, moral, sexuel, etc. ${ }^{2}$. Malgré tout le film a été invité à Cannes et, en raison d'une protestation internationale et brésilienne par voie de presse, le ministre de la Justice a rouvert le procès et a libéré le film sans coupures. Mais quand on l'a diffusé, la plus grande partie de la gauche « officielle » l'a attaqué en l'accusant de fasciste. C'était une énorme polémique sociale, culturelle et politique; aujourd'hui, Terra em transe, deux ans après son lancement, reste d'actualité : la presse s'en occupe, en parle, et le public - aussi bien celui qui a vu le film et ne l'a pas compris que celui qui l'a vu et a réagi contre - a pris conscience de lui. La réalité c'est que le film, maintenant qu'on est train de le relancer et qu'il sera exhibé pendant cinq ans à Rio, São Paulo et dans les états, commence à prendre sa place. Et il a suscité beaucoup de choses au Brésil: il a déterminé une grande influence dans le théâtre, parce qu'ensuite il y a eu un groupe qui a monté $O$ rei da vela, une pièce qui m'a été dédiée et dont l'auteur est Oswald de Andrade, recherche dans l'œuvre de l'un des auteurs de 1922 qui a provoqué un grand scandale dans nos milieux de théâtre. A partir de Terra em transe, Caetano Veloso a initié le mouvement musical tropicaliste. Toute une nouvelle discussion sur la culture brésilienne, spécialement celle engagée, ou plutôt, liée (l'autre terme me déplaît, car démagogique) au sociopolitique, a été posée.

5 Si j'expose ça, ce n'est pas par pure vanité, mais seulement pour expliquer et démontrer une idée valable : que l'intolérance en matière d'art, c'est le pire des crimes qui puisse exister. Parce qu'il se trouve que ceux qu'on appelle les théoriciens de l'art ou les défenseurs d'une école artistique quelconque n'ont pas de souplesse de jugement. Et dans l'art, les seules œuvres qui fonctionnent sont celles de rupture, dans l'art les théories préalables ne valent rien, surtout dans les pays sous-développés, où on ne peut faire de la critique d'art qu'à partir de certains phénomènes donnés, mettant fin à toute théorie préalable, tout préjugé, inévitablement dépourvus de liens. Pour cette raison, Terra em transe a été une expérience vitale pour moi. Et du point de vue international le film a été bien reçu à Cannes et sur le marché européen, il a eu plusieurs prix. Maintenant qu'il sera lancé à Buenos Aires, je suis sûr qu'il continuera à susciter la polémique dans le public d'Amérique latine qui ira le voir. Parce que ça ne m'intéresse pas qu'on dise que c'est un bon ou un mauvais film : dès lors que quelqu'un donne dans son œuvre un niveau de caractère technique et de préoccupation esthétique libérés de primarité et dès lors que le film existe en tant que phénomène cinématographique à l'écran, le problème de le classer bon ou mauvais, beau ou laid, est complètement absurde. L'art d'aujourd'hui n'est plus lié à ce type de concepts.

6 Terra em transe est un film sur ce qui existe de grotesque, de hideux et de pauvre en Amérique latine. Ce n'est pas un film avec des personnages positifs, pas un film de héros parfaits, qui traite du conflit, de la misère, de la pourriture du sous-développé. Pourriture mentale, culturelle, décadence présentes autant dans la droite que dans la gauche. Parce que notre sous-développement, outre les fièvres idéologiques, est de civilisation, provoqué par une oppression économique énorme. Dès lors, nous ne pouvons pas avoir des héros positifs et définis, nous ne pouvons pas adopter des mots de beauté, des mots idéaux. Nous devons affronter notre réalité avec une douleur 
profonde, comme une étude de la douleur. Rien n'existe de positif en Amérique latine à part la douleur, la misère, c'est-à-dire, le positif c'est justement ce qu'on perçoit comme négatif. Parce que c'est à partir de là qu'il est possible de construire une civilisation qui a un long chemin devant elle. C'est mon opinion sur le film.

\section{Cardenas et Capriles : Répercussion nationale et internationale.}

7 Là-dessus j'ai déjà répondu. C'est maintenant que le film est plus actuel. En Argentine le mois prochain, aux Etats-Unis dans trois mois, il suscitera de nouvelles polémiques. Je continue à voir le film comme je l'ai vu avant. Parce que le film n'est pas mort, il continue à agir et je le considère la plus importante de mes oeuvres. La critique conservatrice s'obstine à dire que mon meilleur film est Deus e o diabo. Pour moi, c'est un film académique, romantique, et lié à la culture établie au Brésil. Jusqu'à un certain point, il est traditionaliste, culturaliste. Terra em transe est un film d'observation personnelle, directement lié à ma réalité, sans autre point d'appui dans la tradition culturelle brésilienne. Avec Deus e o diabo, ce qui se passe c'est que, quand il est montré à un public, surtout ce public qui se dit "de gauche", à la fin les spectateurs applaudissent et crient des bravos. Je déteste cette réaction romantique et démagogique. Ce qui intéresse c'est que, dans Terra em transe, un film d'impact par le désordre intellectuel qu'il suscite, oblige les spectateurs à penser, alors que Deus e o diabo provoque leur enthousiasme. J'ai fait Terra em transe contre l'esprit de Deus e o diabo, qui d'une certaine manière est un film conservateur. Ceux qui sont contre Terra em transe disent que "je n'ai pas répété les images de Deus e o diabo ». C'est exactement ce que j'ai voulu faire : ne pas donner la même vision, ne pas faire de démagogie làdessus. Parce que ça ne m'intéresse pas de faire une carrière artistique en tant que cinéaste ni d'atteindre le succès social à partir de mon cinéma. Ce qui m'intéresse c'est de susciter la polémique, participer à une activité culturelle et politique que je fais du mieux que je peux. C'est pourquoi Terra em transe me semble mon film le plus important et c'est celui que je défends le plus, même si je sais qu'il a des défauts. Parce que c'est des défauts qui n'auraient jamais pu cesser d'exister. Si j'avais fait un film sur la transe de l'Amérique latine et que je lui avais donné une forme achevée, je serais en train d'aller à l'encontre de la praxis même du film. Un film de rupture, de crise, doit être aussi pauvre que son propre thème, tout intégré. C'est-à-dire, sans faire de différences entre la forme et le contenu (c'est là une vieille discussion académique); le film est une expression totalisante, névrotique, politique, sociale, personnelle, sexuelle de tout ce que, en tant que latino-américain de 31 ans, je peux exprimer en vivant cette réalité et en exerçant une activité radicale par rapport à elle, par rapport à ma façon de l'exprimer. (...)

\section{Et O D ragão da maldade contra o santo guerreiro ?}

8 C'est lié à mes autres films. Antônio das Mortes est un personnage de Deus e o diabo, mais l'optique est différente. Premièrement : ce n'est pas un film romantique, il n'a pas la dose de romantisme de Deus e o diabo ; c'est un film qui apporte une maturation des personnages en ce qui concerne Terra em transe. Deuxièmement: de ce point de vue esthétique, c'est un film sans l'agitation de Terra em transe et sans l'intellectualisme qui existe dans Deus e o diabo. C'est un film moderne comparativement aux autres et avec l'intention d'obtenir une communication plus grande, plus directe, avec le public. 
9 C'est une expérience nouvelle pour moi, car c'est un film que j'ai fait après avoir compris que certaines choses dont j'avais parlé dans mes œuvres, que certains témoignages et obsessions intérieures n'intéressaient pas réellement le public et se donnaient sur un terrain littéraire, mon cinéma n'étant pas encore mûr pour l'exposition de ce type de problèmes. J'ai fait $O$ Dragão en cherchant à simplifier pour le grand public une série de problèmes complexes et, même ceux-là, en des termes les plus simples possibles. Ce que je dis peut paraître compliqué; c'est présenter un panorama plus ouvert, spontané, sans romanticismes et en supprimant les personnages intellectuels de mes autres films. Ici les personnages sont un peu à distance par rapport à moi, parce que dans Deus e o diabo même Corisco est un personnage intellectuel qui dit des choses que je pense, une sorte de vecteur de mes réflexions philosophiques. Pas dans $O$ Dragão. C'est des personnages épiques, des personnages de western entièrement libres dans leur action et filmés d'une façon très froide ; participante et froide en même temps. C'est difficile d'expliquer ça. C'est un film dont je ne peux pas encore parler, et, comme il n'a pas encore été diffusé, je ne l'ai pas encore discuté.

Le cinéma est un art qui doit se communiquer, si cela n'arrive pas il n'y a pas de sens à en faire : ça reste un produit technologique du XX siècle destiné à être absorbé par la télé, une vieille chose qui ne trouve même pas de salle de projection. Le cinéaste moderne aujourd'hui est celui qui peut communiquer les problèmes les plus complexes et les plus profonds à travers un langage qui soit compris. Ce langage n'est plus celui du cinéma américain, langage dictatorial qui doit être réformé et brisé. Il y a un autre type de langage communicatif qui peut s'appliquer. O Dragão en est une tentative. Il a un esprit de western traditionnel dans le climat brésilien qui est le même de Deus e o diabo. Avec des constatations culturelles plus nettes. Différent de Terra em transe, qui est un film qui se déroule dans un milieu bourgeois, typique de certaines métropoles d'Amérique latine. O Dragão a pour scène un milieu paysan typiquement brésilien.

11 Je suis sûr que ce film connaîtra un grand succès auprès du public. Et que la critique esthéticienne et préjudiciable dira à nouveau que j'ai abandonné les recherches de Terra em transe. Cela n'a pas d'importance, de même que ce qui a été dit de Deus e o diabo n'en a pas eue non plus. J'ai fait ce film comme on fait une expérience et le prochain ne lui ressemblera pas. D'ailleurs, c'est une expérience que je fais dans le cinéma latinoaméricain, mais sans intention d'ouvrir la voie à qui que ce soit, parce que je crois que tout cinéaste a sa propre voie. Un critique écrivait que mes films sont des voies pour le cinéma d'Amérique latine. Je suis contraire à ça et ça me semble absurde, de même que lorsqu'on dit que je suis le leader, le porte-parole, le théoricien du cinema novo ... Je ne suis rien; le cinema novo est un mouvement d'organisation et d'action, de production économique. C'est un diffuseur qui s'appelle DIFILM et un autre qui s'appelle MAPA FILMES. Culturellement, chaque cinéaste fait le sien, les films du cinema novo sont entièrement différents les uns des autres. Evidemment, nous, les cinéastes qui formons le mouvement, nous avons des points de vue communs d'intérêt esthétique, artistique ou culturel, mais les films de chacun sont différents. Et il n'y a pas telle ou telle tendance dominante. Je ne suis le porte-parole d'aucune théorie. Je cherche mon chemin comme, je crois, n'importe quel cinéaste latino-américain complexe et diversifié. [...] 


\section{Cancer.} qui se passe c'est que chacun a sa forme. En peinture, des fois on décide de faire une fresque murale, d'autres un petit tableau. Cela ne veut pas dire que la fresque murale soit plus importante pour autant. Tout est lié à l'expérience culturelle, existentielle, humaine de l'auteur. Câncer est un film pour lequel ça n'avait pas de sens de tourner en couleurs ou en $35 \mathrm{~mm}$. Ce n'est pas un film commercial, je ne l'ai pas fait pour être exhibé dans un circuit. C'est une œuvre avec laquelle je me suis amusé avec mes amis. J'ai décidé de faire le film en $16 \mathrm{~mm}$, j'ai appelé mes acteurs, mes amis, et je leur ai dit : « Nous allons faire un film ». J'ai fait et ça ne m'a rien coûté, le matériel est là, mais il n'est pas prêt et je ne sais pas quand je vais le préparer. J'ai fait le film aussi pour démontrer que dans le cinéma il n'y a pas un seul chemin. J'ai filmé Câncer quelques jours avant de commencer $O$ Dragão parce que le tournage avait pris du retard et j'allais rester un mois à rien faire. J'ai fait appel à deux acteurs de $O$ Dragão et j'ai fait un essai avec prise de son directe. A cette époque là, certains disaient : « Le chemin du cinéma, c'est le film en couleurs, grand spectacle » et d'autres : «Le chemin du cinéma, c'est le film de $16 \mathrm{~mm}$, underground ». préoccupations, mais tout aussi liées à ma personne que celles de $O$ Dragão. C'est des films différents et semblables en même temps. Câncer est un film particulier, je ne l'enverrai pas à des festivals, pas plus que je ne le diffuserai dans les cinémas. Ou peutêtre si, mais je ne l'ai pas encore terminé, il manque le montage. En ce moment, ça ne m'intéresse pas de le faire parce que mon plaisir c'était juste de le filmer et je suppose que peut-être ce qui est dedans n'a pas d'importance.

Le film n'a pas d'histoire. C'est trois personnages dans une action violente. Ce que je cherchais, c'était à faire une expérience technique, du problème de la résistance de la durée du plan cinématographique. On y voit comment la technique intervient dans le processus cinématographique.

Je m'explique : comme je savais que l'Eclair a un châssis qui donne de 11 à 12 minutes, j'ai décidé de faire un film où chaque plan aurait la durée d'un châssis, et étudier la quasi élimination du montage lorsqu'il existe une action verbale et psychologique constante dans la même prise. Parce que de même que le théâtre moderne est en train d'amener le cinéma aux peuples, le cinéma doit aussi être porteur de beaucoup de théâtre. C'est mon point de vue, ce n'est pas une théorie pour qui que ce soit. C'est mon expérience. J'utilise le théâtre dans mes films de façon délibérée. Pour cette raison, je ne ferai jamais de mise en scène, j'en fais dans mes films, et ça me plaît. De même que j'utilise le théâtre, j'utilise l'opéra, je trouve que tout ça fonctionne pour ce que je veux exprimer. Ce qui ne veut pas dire que je ne pourrai pas faire autre chose demain. C'est pour ça que j'aime le film de Gustavo Dahl 0 bravo guerreiro, expression parfaite de la pensée : rationnel, sec. Je n'aime pas le cinéma baroque, j'en fais, mais j'aime des films 
différents du mien; celui de Straub, par exemple : sa Chronique d'Anna Magdalena Bach me semble un chef d'œuvre. Autrement dit, je ne préfère pas les films qui sont sur ma ligne cinématographique. Ce n'est pas le cas pour moi.

Pour revenir à Câncer. J'avais beaucoup discuté avec Straub à Berlin sur le thème du plan/séquence et j'ai décidé de faire des expériences à partir desquelles Straub est en train de faire. Car Straub est le premier à expérimenter sur ce terrain; bon, non pas le premier - Hitchcock l'avait déjà fait mais c'était autre chose - même si ce que Straub cherche à obtenir c'est une tension, un sens nouveau du plan/séquence. Bien sûr, Godard est en train de le faire aussi, de même que le cinéma underground américain. Mais l'expérience était nouvelle pour moi, non pas nouvelle pour le cinéma puisqu'on a déjà tout fait.

20 J'ai fait Câncer comme ça. C'est-à-dire : 27 plans longs et trois acteurs qui improvisaient des situations dont le thème - que je leur donnais - était celui de la violence. Violence psychique, sexuelle, raciale, tout ça sur un plan d'improvisation. Maintenant il reste à monter, pour voir les résultats. Je ne pouvais faire ça qu'avec une Eclair $16 \mathrm{~mm}$. Si je l'avais fait en $35 \mathrm{~mm}$, ça n'aurait intéressé personne. Et ça m'aurait coûté beaucoup, pour n'intéresser personne d'autre que des critiques et des groupes fermés ou la diffusion dans des cinéclubs. Sans aucun intérêt pour le grand public. [...]

\section{Expérience brésilienne}

21 Ce que nous cherchons à prouver au Brésil, c'est qu'il est possible de créer du cinéma dans un pays sous-développé. Et que notre expérience puisse servir aux autres pays d'Amérique latine. Mais je suis allé à Buenos Aires et les gens là-bas ne comprennent pas, ils sont individualistes, prisonniers d'un concept anticinématographique, et ceux qui dénoncent l'aliénation économique et politique sont aliénés devant le cinéma.

Ce n'est pas comme ça que ça marche. Il faut que les jeunes cinéastes d'Amérique latine, les producteurs, s'unissent entre eux. Nous avons voulu conquérir le marché européen parce que cela signifiait des dollars et leur entrée au Brésil pour faire plus de films. Indépendamment du fait que notre succès au niveau européen aide, en interne, à briser le préjugé de notre public vis-à-vis de l'art et du cinéma brésilien. C'était notre mécanique politique.

Le cinema novo, c'est ça - une organisation économique structurée; un film peut échouer, même deux ou dix, mais le cinema novo ne va pas échouer parce que nous sommes organisés et c'est cela qui compte. Si je fais un film mauvais, si Saraceni, un bon ou si Joaquim Pedro un mauvais, ça n'a pas d'importance. L'important, c'est le cinema novo et le cinéma brésilien, dont nous sommes 35 à $40 \%$ de sa production. Ce que nous voulons c'est que le cinema novo soit le cinéma brésilien : en communiquant avec le public, en ayant son propre marché, en exportant. Que le cinema novo soit industrie, avec une nouvelle mentalité, où le réalisateur puisse avoir une liberté de création, où le producteur soit technicien en économie, où la création puisse se développer à un rythme sain, où les enjeux commerciaux ne relèvent pas de l'usure mais d'une proportion d'intérêt administrativement calculée; bref: une industrie moderne capable de bien fonctionner aujourd'hui et demain, et sous le régime politique qu'il y aura au Brésil. Nous voulons créer pour l'avenir une tradition esthétique et économique de cinéma. Nous ne l'avons pas encore, mais nous voulons la créer et qu'elle soit 
maintenue et respectée par-dessus n'importe quel dogmatisme idéologique qui puisse exister au Brésil. C'est notre politique, c'est pour ça que nous luttons.

Si on construit une industrie et un art du cinéma au Brésil, étant donnée l'importance du cinéma dans le monde moderne - et il en aura de plus en plus dans l'avenir, diffusé par la télé et créé à travers des techniques modernes - ce sera notre façon directe d'agir dans le processus de lutte contre le sous-développement. Y compris parce que du point de vue politique, je crois que dans la pensée révolutionnaire latino-américaine il faut remplacer immédiatement une vieille terminologie moscovite viciée : le mot, c'est lutte contre le sous-développement. Ce que nous devons atteindre, c'est la productivité développementiste, dans tous les secteurs: politique, social, technique, tout. Alors, l'action politique révolutionnaire du cinéma brésilien est favorable à cette action à créer. Parce que le cinéma ne peut être instrument de collaboration profonde dans la lutte pour le développement, pour l'émancipation brésilienne, que si - avant toute chose - il existe. Notre concentration, et celle de tout cinéaste d'Amérique latine, doit porter là-dessus : le cinéma ne devient un instrument puissant de communication et de modification de la pensée des masses que lorsqu'il parvient à être communiqué. Et ce n'est pas un seul film qui va y arriver. C'est une cinématographie, c'est la maîtrise d'un marché. Parce que les films, pour qu'ils se communiquent, il faut qu'ils soient diffusés et, pour cela, ils ont besoin d'une salle de projection ou d'une chaîne de télé.

\section{Télévision}

25 Je crois, même, qu'il n'existe aucun conflit entre cinéma et télévision. Le conflit peut exister entre la salle de projection et le petit écran, mais pour le cinéaste c'est la même chose, puisqu'il fait son film de la même manière, et ce dernier ira, soit à la télévision, soit au cinéma. Et il devrait aller d'abord à la télévision et ensuite au cinéma. C'est fondamentalement pratique. Mais le cinéaste est prisonnier du préjugé qui veut que son film soit projeté dans un cinéma. Être soumis au rite "sacré »: un individu doit arriver, acheter l'entrée, s'asseoir, voir le film sur grand écran, et le journal publier un commentaire dans sa section de critique hebdomadaire. Tout ça, ce ne sont que des préjugés, le cinéma n'est plus un rituel, mais quelque chose de profane; le film doit passer à la télé et ensuite il pourra ou non passer au cinéma. Les grands films, comme les grandes œuvres de la peinture, continueront à passer dans les cinémas - musées ou salles d'art - c'est ainsi avec Le cuirassé Potemkine, qui sera toujours plus vu que James Bond, car il continue à être exhibé depuis plus de trente ans et s'il y a deux millions qui voient Bond en un mois, il y a dix millions qui ont vu Le cuirassé pendant toutes ces années. A la télé européenne, on le donne tout le temps.

Il faut que nous prenions en compte cela : le fait que Le cuirassé soit passé à la télé n'est pas déshonorant pour le film, au contraire. Quand Vidas Secas, de Nelson ${ }^{3}$, a été projeté à la télé au Brésil, c'est là que le film s'est réalisé. Parce qu'à Rio deux millions de personnes ont vu le film et, même si elles ne savaient pas qu'il était de Nelson, que c'était du cinema novo, rien, elles l'ont $v u$, et c'est ce qui importe. Il faut en finir avec le rituel cinématographique. Le cinéma doit exister aujourd'hui, au-delà de l'information, de la polémique et de la discussion. Dans les pays sous-développés, une façon de résoudre le problème de la distribution, c'est de faire que les films qu'on ne voit pas soient lancés par la télé. Parce que nous n'allons pas passer toute notre vie à lutter 
contre elle, et il faudra attendre encore des années et des années avant qu'on puisse mettre en place une chaîne de diffusion.

Si vous commencez maintenant au Pérou, quand vous y parviendrez, vous serez déjà en train de vivre pleinement l'ère de l'apogée de la télé. Ce qui doit être fait alors, c'est profiter de la télé, culturelle ou commerciale, et exhiber les films qui seront faits. Par conséquent, monter une structure de distribution-exhibition pour la consommation directe et la préservation de la culture cinématographique ou disparaître. Pour obtenir de la communication de masse, il faudra utiliser la télé. Pour cette raison mon film 0 Dragão a été fait dans un schéma de télévision. J'ai vendu préalablement le film à la télé allemande, et il sera lancé par la télé avant les cinémas d'Allemagne et de France. Ainsi, en une seule fois, neuf millions de personnes en Allemagne et huit millions en France vont le voir. Comment pourrais-je obtenir un tel volume de public à moins d'avoir la chaîne de distribution-exhibition américaine à mon service ? Le film ira après dans les salles de projection et le spectateur qui voudra le voir sur grand écran pourra le faire. Il faut que nous ayons ça en tête : l'importance de la télé.

Mais le cinéaste d'Amérique latine veut encore aller aux festivals, paraître dans les Cahiers ${ }^{4}$, toutes ces idées sous-développées propres aux littéraires du XIX siècle. Il s'agit d'autre chose : profiter de la technologie le mieux possible pour obtenir la meilleure communication possible.

\section{Esthetique de la faim ${ }^{5}$ (eztetyka da fome)}

Thèse présentée durant les discussions autour du cinema novo, à l'occasion de la rétrospective réalisée à la V Rassegna del Cinema Latino-Americano, à Gênes, janvier 1965, sous le parrainage du Columbianum. Le thème proposé par le secrétaire Aldo Viganò a été Cinema novo et cinéma mondial. Des contingences ont forcé la modification : le paternalisme de l'Européen vis-à-vis du Tiers Monde a été le principal motif du changement de ton.

En me dispensant de l'introduction informative, devenue la caractéristique générale des discussions sur l'Amérique latine, je préfère situer les réactions entre notre culture et la culture civilisée dans des termes moins réduits que ceux qui, aussi, caractérisent l'analyse de l'observateur européen. Ainsi, tandis que l'Amérique latine se plaint de ses misères générales, l'interlocuteur étranger cultive la saveur de cette misère, non pas comme symptôme tragique, mais seulement comme donnée formelle en son champ d'intérêt. Ni le Latin ne communique sa véritable misère à l'homme civilisé, ni l'homme civilisé ne comprend véritablement la misère du Latin.

31 Voici - fondamentalement - la situation des Arts au Brésil devant le monde : jusqu'à aujourd'hui, seuls des mensonges élaborés de la vérité (les exotismes formels qui jugeraient des problèmes sociaux) ont pu être communiqués en termes quantitatifs, provoquant une série d'équivoques qui ne s'arrêtent pas aux frontières de l'Art mais contaminent surtout le terrain général du politique.

Pour l'observateur européen, les processus de création artistique du monde sousdéveloppé ne l'intéressent que dans la mesure où ils satisfont sa nostalgie du primitivisme ; et ce primitivisme se présente hybride, déguisé sous des héritages tardifs du monde civilisé, mal compris car imposés par le conditionnement colonialiste.

33 L'Amérique latine demeure colonie et ce qui différencie le colonialisme d'hier de l'actuel, c'est seulement la forme plus perfectionnée du colonisateur : et au-delà des 
colonisateurs de fait, les formes plus subtiles de ceux qui contre nous aussi préparent de futures attaques.

Le problème international de l'Amérique latine est encore un cas de changement de colonisateurs, alors qu'une libération possible sera encore longtemps fonction d'une nouvelle dépendance.

Ce conditionnement économique et politique nous a conduit au rachitisme philosophique et à l'impuissance, qui, parfois inconsciente, parfois non, engendrent dans le premier cas la stérilité et dans le second, l'hystérie.

La stérilité : ces œuvres qu'on trouve abondamment dans nos arts, où l'auteur se châtre dans des exercices formels, qui, toutefois, n'atteignent pas la pleine possession de leurs formes. Le rêve frustré d'universalisation: des artistes qui ne se sont pas réveillés de l'idéal esthétique adolescent. Ainsi, nous voyons des centaines de tableaux dans les galeries, poussiéreux et oubliés ; des livres de contes et poèmes; des pièces de théâtre, des films (qui, surtout à São Paulo, ont provoqué même des faillites)... Le monde officiel chargé des arts a généré des expositions carnavalesques dans plusieurs festivals et biennales, des conférences fabriquées, des formules faciles de succès, des cocktails dans diverses parties du monde, en plus de quelques monstres officiels de la culture, des académiciens de Lettres et Arts, des jurys de peinture et des marches culturelles à travers tout le pays. Des monstruosités universitaires : les fameuses revues littéraires, les concours, les titres.

L'hystérie: un chapitre plus complexe. L'indignation sociale provoque des discours flamboyants. Le premier symptôme en est l'anarchisme qui marque la jeune poésie jusqu'à présent (et la peinture). Le deuxième, c'est une réduction politique de l'art qui fait de la mauvaise politique pour cause d'excès de sectarisme. Le troisième, et le plus efficace, c'est la quête d'une systématisation pour l'art populaire. Mais l'erreur dans tout ça est que notre possible équilibre ne résulte pas d'un corps organique, mais d'un effort titanesque et auto dévastateur dans le sens de surmonter l'impuissance et, dans le résultat de cette opération au forceps, nous nous voyons frustrés, à peine placés immédiatement en dessous du colonisateur : et s'il nous comprend, alors, ce n'est pas par la lucidité de notre dialogue mais par l'humanitarisme que notre information lui inspire. Une fois de plus le paternalisme est la méthode de compréhension pour un langage de larmes ou de souffrance muette.

38 La faim latine, pour cela, n'est pas seulement un symptôme alarmant : c'est le nerf de sa propre société. Là réside l'originalité tragique du cinema novo devant le cinéma mondial : notre originalité est notre faim et notre plus grande misère vient de ce que cette faim, étant ressentie, n'est pas comprise.

D 'Aruanda à Vidas Secas, le cinema novo a narré, décrit, poétisé, mis en discours, analysé, excité les thèmes de la faim: des personnages mangeant de la terre, des personnages mangeant des racines, des personnages volant pour manger, des personnages s'enfuyant pour manger, des personnages sales, laids, décharnés, habitant des maisons sales, laides, sombres ; c'est cette galerie de faméliques qui a identifié le cinema novo au misérabilisme, tellement décrié par le Gouvernement, par la critique au service des intérêts antinationaux, par les producteurs et par le public - ce dernier ne supportant pas les images de sa propre misère. Ce misérabilisme du cinema novo s'oppose à la tendance du digestif préconisée par le Grand Critique de Guanabara, Carlos Lacerda ${ }^{6}$ : des films de gens riches, dans de jolies maisons, circulant dans des automobiles de luxe; des films joyeux, drôles, rapides, dépourvus de messages, ayant des objectifs 
purement industriels. Ce sont des films qui s'opposent à la faim, comme si, sous serre dans les appartements de luxe, les cinéastes pouvaient cacher la misère morale d'une bourgeoisie indéfinie et fragile, ou comme si même les propres matériaux techniques et scénographiques pouvaient cacher la faim qui se trouve enracinée dans la propre incivilisation. Comme si, surtout, dans cet apparat de paysages tropicaux, il était possible de déguiser l'indigence mentale des cinéastes qui font ce type de film. Ce qu'a fait du cinema novo un phénomène d'importance internationale, c'est justement son haut niveau d'engagement vis-à-vis de la vérité ; c'est son propre misérabilisme qui, auparavant écrit par la littérature de 30 , a été désormais photographié par le cinéma de 60 ; et s'il était auparavant écrit comme dénonciation sociale, il est désormais discuté en tant que problème politique. Les stades même du misérabilisme dans notre cinéma sont internement évolutifs. Ainsi, comme l'observe Gustavo Dahl, ça va du phénoménologique (Porto das Caixas), au social (Vidas Secas), au politique (Deus e o diabo), au poétique (Ganga Zumba, rei dos Palmares), au démagogique (Cinco vezes favela), à l'expérimental (Sol sobre a lama), au documentaire (Garrincha, alegria do povo), à la comédie (Os mendigos), expériences allant dans divers sens, frustrées les unes, réalisées les autres, mais toutes composant, au bout de trois années, un tableau historique lequel, non pas par hasard, ira caractériser la période Janio-Jango ${ }^{7}$ : la période des grandes crises de conscience et de rébellion, d'agitation et révolution qui a culminé dans le Coup d'Avril. Et c'est à partir d'Avril que la thèse du cinéma digestif a gagné du poids au Brésil, menaçant, systématiquement, le cinema novo.

Nous comprenons cette faim que l'Européen et le Brésilien en majorité ne comprennent pas. Pour l'Européen, c'est un étrange surréalisme tropical. Pour le Brésilien, c'est une honte nationale. Il ne mange pas mais il a honte de le dire; et, surtout, il ne sait d'où elle vient, cette faim. Nous savons - nous qui avons fait ces films laids et tristes, ces films criés et désespérés où la raison n'a pas toujours parlé plus haut - que la faim ne sera pas guérie par les planifications de cabinet et que les raccommodages du technicolor ne cachent pas mais en aggravent les tumeurs. Ainsi, seule une culture de la faim, minant ses propres structures, peut se surmonter du point de vue qualitatif : et la plus noble des manifestations culturelles de la faim, c'est la violence.

41 La mendicité, une tradition qui s'est implantée avec la pitié rédemptrice colonialiste, a été de tout temps une des causes de la mystification politique et du mensonge culturel nationaliste: les rapports officiels sur la faim demandent de l'argent aux pays colonialistes dans le but de construire des écoles sans créer des enseignants, de construire des maisons sans donner du travail, d'enseigner le métier sans enseigner l'analphabète. La diplomatie demande, les économistes demandent, la politique demande ; le cinema novo, dans le champ international, n'a rien demandé : s'est imposée la violence de ses images et sons dans 22 festivals internationaux.

42 Par le cinema novo: le comportement exact d'un famélique, c'est la violence, et la violence d'un famélique, ce n'est pas du primitivisme. Fabiano est-il primitif? Antão est-il primitif ? Corisco est-il primitif ? La femme de Porto das Caixas est-elle primitive ?

Du cinema novo : une esthétique de la violence, avant d'être primitive est révolutionnaire, voici un point de départ pour que le colonisateur comprenne l'existence du colonisé ; seulement en conscientisant sa possibilité unique, la violence, le colonisateur peut comprendre, par l'horreur, la force de la culture qu'il exploite. Tant qu'il n'a pas levé les armes le colonisé est un esclave : il fallut un premier policier tué pour que le Français aperçût un Algérien. 

novo nécessite de s'effectuer pour s'expliquer au fur et à mesure que notre réalité deviendra plus discernable à la lumière de pensées qui ne soient pas affaiblies ou délirantes par la faim. Le cinema novo ne peut se développer effectivement, tant qu'il restera en marge du processus économique et culturel du continent latino-américain ; y compris parce que le cinema novo est un phénomène des peuples colonisés et non pas une entité privilégiée du Brésil : là où il y aura un cinéaste prêt à filmer la vérité et à affronter les canons hypocrites et policiers de la censure, là il y aura un germe vivant du cinema novo. Là où il y aura un cinéaste prêt à affronter la marchandisation, l'exploitation, la pornographie, le technicisme, là il y aura un germe du cinema novo. Là où il y aura un cinéaste, peu importe son âge ou son origine, prêt à mettre son cinéma et sa profession au service des causes importantes de son temps, là il y aura un germe du cinema novo. C'est ça la définition et en raison de cette définition le cinema novo se marginalise de l'industrie, parce que la compromission du cinéma industriel, c'est par rapport au mensonge et à l'exploitation. L'intégration économique et industrielle du cinema novo dépend de la liberté de l'Amérique latine. Pour cette liberté, le cinema novo s'engage, en son nom propre, de ses membres le plus proches et les plus dispersés, des plus bornés aux plus talentueux, des plus faibles aux plus forts. C'est une question de morale qui se reflétera dans les films, dans le temps consacré à filmer un homme ou une maison, dans le détail observé, dans la Philosophie: ce n'est pas un film mais un ensemble de films en évolution qui, au final, donnera au public la conscience de sa propre existence. cela même, de toutes les faiblesses conséquentes de son existence.

\section{Esthétique du rêve ${ }^{8}$ (eztetyka do sonho)}

Lors du Séminaire du Tiers Monde, réalisé à Gênes, Italie, 1965, j'ai présenté, à propos du cinema novo brésilien, « L'esthétique de la faim ». 
Cette communication situait l'artiste du Tiers Monde face aux puissances colonisatrices: seule une esthétique de la violence pourrait intégrer une signification révolutionnaire dans nos luttes de libération.

Il était dit que notre pauvreté était comprise mais jamais ressentie par les observateurs coloniaux.

1968 a été l'année des rébellions de la jeunesse.

Le mai français est survenu à un moment où des étudiants et des intellectuels brésiliens manifestaient au Brésil leur protestation contre le régime militaire de 1964.

Terre en transe, 1966, un manifeste pratique de l'esthétique de la faim, a subi au Brésil des critiques intolérantes de la droite et des groupes sectaires de la gauche.

Entre la répression interne et la répercussion internationale, j'ai appris la meilleure des leçons : l'artiste doit maintenir sa liberté en toute circonstance.

Seulement ainsi nous serons libérés d'un type très original d'appauvrissement: l'officialisation à laquelle les pays sous-développés ont coutume de soumettre leurs meilleurs artistes.

7 Ce Congrès à Columbia est une autre opportunité dont je dispose pour développer quelques idées à propos d'art et révolution. Le thème de la pauvreté est lié à ça.

Les Sciences Sociales ont informé des statistiques et permettent des interprétations sur la pauvreté.

Les conclusions des rapports des systèmes capitalistes envisagent l'homme pauvre comme un objet qui doit être nourri. Et dans les pays socialistes nous observons la polémique permanente entre les prophètes de la révolution totale et les bureaucrates qui traitent l'homme comme un objet à massifier. La plupart des prophètes de la révolution totale est composée d'artistes. Ce sont des personnes qui ont une approche plus sensible et moins intellectuelle des masses pauvres.

60 Art révolutionnaire a été le mot d'ordre dans le Tiers Monde dans les années 60 et ça va continuer en cette décennie. Je crois, cependant, que le changement de beaucoup de conditions politiques et mentales exige un développement continu des concepts d'art révolutionnaire.

61 Primarité souvent se confond avec les manifestes idéologiques. Le pire ennemi de l'art révolutionnaire est sa médiocrité. Devant l'évolution subtile des concepts réformistes de l'idéologie impérialiste, l'artiste doit offrir des réponses révolutionnaires capables de n'accepter, en aucune hypothèse, les esquives proposées. Et, ce qui est le plus difficile, cela exige une identification précise de ce qu'est l'art révolutionnaire utile à l'activisme politique, de ce qu'est l'art révolutionnaire lancé dans l'ouverture de nouvelles discussions de ce qu'est l'art révolutionnaire rejeté par la gauche et instrumentalisé par la droite.

2 Dans le premier cas je cite, en tant qu'homme de cinéma, le film de Fernando Ezequiel Solanas, argentin, La hora de los hornos. C'est typiquement un pamphlet d'information, agitation et polémique actuellement utilisé dans diverses parties du monde par des activistes politiques.

Dans le deuxième cas j'ai quelques films du cinema novo brésilien parmi lesquels mes propres films.

64 Et en dernier l'œuvre de Jorge Luis Borges. 
Cette classification révèle les contradictions d'un art exprimant le propre cas contemporain. Une œuvre d'art révolutionnaire devrait non seulement agir sur un mode immédiatement politique mais aussi promouvoir la spéculation philosophique, en créant une esthétique de l'éternel mouvement humain vers son intégration cosmique.

L'existence discontinue de cet art révolutionnaire dans le Tiers Monde se doit fondamentalement aux répressions du rationalisme.

Les systèmes culturels agissants, de droite et de gauche, sont attachés à une raison conservatrice. L'échec des gauches au Brésil résulte de ce vice colonisateur. La droite pense selon la raison de l'ordre et du développement. La technologie est l'idéal médiocre d'un pouvoir qui n'a d'autre idéologie que la domination de l'homme par la consommation. Les réponses de la gauche, j'exemplifie une fois de plus au Brésil, ont été paternalistes par rapport au thème central des conflits politiques: les masses pauvres.

Le peuple est le mythe de la bourgeoisie.

La raison du peuple devient la raison de la bourgeoisie sur le peuple.

Les variations idéologiques de cette raison paternaliste s'identifient dans des cycles monotones de protestation et répression. La raison de gauche révèle l'héritier de la raison révolutionnaire bourgeoise européenne. La colonisation, à un tel niveau, rend impossible une idéologie révolutionnaire intégrale qui aurait dans l'art son expression majeure, parce que seul l'art peut approcher l'homme avec la profondeur que le rêve de cette compréhension puisse permettre.

1 La rupture avec les rationalismes colonisateurs est la seule issue.

Les avant-gardes de la pensée ne peuvent plus s'adonner au succès inutile consistant à répondre à la raison oppressive avec la raison révolutionnaire. La révolution est l'antiraison qui communique les tensions et rébellions du plus irrationnel de tous les phénomènes qu'est la pauvreté.

Aucune statistique ne peut informer la dimension de la pauvreté.

La pauvreté est la charge autodestructive maximale de chaque homme et elle répercute psychiquement d'une telle façon que ce pauvre se convertit en animal à deux têtes: l'une est fataliste et soumise à la raison qui l'exploite en tant qu'esclave. L'autre, dans la mesure où le pauvre ne peut expliquer l'absurdité de sa propre pauvreté, est naturellement mystique.

75 La raison dominatrice classifie le mysticisme d'irrationaliste et le réprime par balle. Pour elle tout ce qui est irrationnel doit être détruit, que ce soit la mystique religieuse, ou la mystique politique. La révolution, en tant que possession de l'homme qui lance sa vie en direction d'une idée, est l'expression la plus heureuse du mysticisme. Les révolutions échouent quand cette possession n'est pas totale, quand l'homme rebelle ne se libère pas totalement de la raison répressive, quand les signes de la lutte ne se produisent à un niveau d'émotion stimulante et révélatrice, quand, mû encore par la raison bourgeoise, méthode et idéologie se confondent à un tel point qu'ils paralysent les transactions de la lutte.

Dans la mesure où la déraison planifie les révolutions, la raison planifie la répression.

Les révolutions se font dans l'imprévisibilité de la pratique historique qui est la cabale de la rencontre des forces irrationnelles des masses pauvres. La prise politique du pouvoir n'implique pas le succès révolutionnaire. 

Ce mysticisme est le seul langage qui transcende le schéma rationnel de l'oppression. La révolution est une magique parce que c'est l'imprévu à l'intérieur de la raison dominatrice. Au mieux elle vue comme une possibilité compréhensible. Mais la révolution doit être une impossibilité de compréhension pour la raison dominatrice de telle façon qu'elle en vienne à se nier elle-même et à se dévorer face à son impossibilité de comprendre. même lors des rencontres de la violence provoquée par le système, signifie toujours nier la violence au nom d'une communauté fondée par le sens de l'amour illimité entre les hommes. Cet amour n'a rien à voir avec l'humanisme traditionnel, symbole de la bonne conscience dominatrice.

Les racines indiennes et noires du peuple latino-américain doivent être comprises comme seule force développée de ce continent. Nos classes moyennes et bourgeoisies sont des caricatures décadentes des sociétés colonisatrices. avec le thème de mes films, sens naturel de ma vie.

89 Columbia University - New York. Janvier, 1971

\section{« L'art comme laboratoire d'expérimentation des conflits $»-$ par Ismail XAVIER ${ }^{9}$}

Glauber Rocha émerge en tant que figure marquante du cinema novo ${ }^{10}$ au tournant des années 1950 et 1960, et il incarne bien une génération d'intellectuels et d'artistes 
marqués par une conscience historique aiguë, toujours attentive au lien entre culture et politique. Les paramètres de sa création se sont définis alors que le pays vivait une transition décisive entre la politique «développementiste» du gouvernement de Juscelino Kubitschek et la polarisation des conflits sociaux du début des années 1960, période marquée par de graves crises politiques. C'est à cette époque, avec pour arrière fond les luttes autour d'un certain nombre de réformes sociales et de projets nationalistes de gauche, que se dessine l'horizon de son œuvre, composée de nombreux films mais aussi d'un vaste ensemble d'articles ${ }^{11}$, et sujette pour le reste à des variations liées à la manière par laquelle il répond à chaque nouvelle conjoncture, brésilienne et mondiale.

De Barravento (1961) à L'âge de la terre (1980), son cinéma affirme sa veine politique et de renouvellement esthétique, élargissant peu à peu le terrain sur lequel il situe son débat sur l'histoire et la conscience des hommes, un débat fait d'articulations entre misère économique et expérience religieuse, lutte des classes et imagination politique. Son cinéma part d'une plage isolée avec sa communauté de pêcheurs (Barravento); plonge ensuite dans l'expérience paysanne, revient sur l'histoire de la campagne aride (le "sertão ") et sa tradition de misère et de violence (Deus e o diabo na terra do sol) ${ }^{12}$; approche ensuite, dans Terre en transe, les abords d'un pays imaginaire, Eldorado, allégorie non seulement du Brésil mais de l'Amérique latine et de sa politique, détachée de la crise des projets de libération nationale mis en échec par une série de coups d'Etat militaires. De l'Amérique latine, il va vers l'Afrique (Le lyon à sept têtes) et en Europe (Espagne, Têtes coupées, et Italie, Claro).

Penser son cinéma et ses textes critiques, c'est s'arrêter sur son interrogation - large, ambitieuse - adressée à notre temps à partir de l'optique du Tiers Monde (expression emblématique à l'époque où Glauber réalise son œuvre). Son point focal s'est toujours porté sur les questions collectives, pensées à grande échelle, à travers une scène où les personnages de ses films et les personnalités évoquées dans ses textes se présentent comme autant de condensations de l'expérience de groupes, de classes, de nations. La vie sociale, dans son cinéma, est conçue comme une expérience de crises et de ruptures, faite d'actes de domination et de résistance, de journées de l'opprimé en quête de justice. Il y a un désir d'histoire, une attention portée au déséquilibre, un jeu de forces, un dynamisme qui demande une figuration dramatique à la hauteur et un style capable d'absorber les tensions mises en scène, autant dans les films que dans l'écriture. Une telle figuration, le cinéma de Glauber l'a recherchée à travers la cristallisation du mouvement du monde dans des métaphores capables de fournir l'image globale de la crise vécue. Une telle perception totalisante s'est tantôt configurée dans la force convulsive du vent et des marées (Barravento), tantôt dans la prophétie révolutionnaire - «le sertão (la campagne aride) deviendra la mer, et la mer deviendra sertão » (Deuse o diabo na terra do sol) -, tantôt dans la «transe " qui s'empare de la vie politique d'Eldorado et contamine toutes les forces en conflit au moment du coup d'Etat (Terre en transe).

Après le Festival de Cannes de 1969, lorsqu'il obtient le prix de meilleur directeur, avec 0 dragão da maldade contra o santo guerreiro ${ }^{13}$, il a pu rendre effective une pratique traduisant son modèle de "cinéaste tricontinental", et devenant par là même une personnalité importante du débat culturel et politique de l'époque. Un engagement militant intense et des luttes multiples ont ponctué une période de migrations où, en France comme en République du Congo, en Espagne, à Cuba ou en Italie, il a filmé, écrit, 
accordé des entretiens et s'est engagé dans des polémiques, se battant toujours pour maintenir en vie le projet d'un cinéma d'auteur marqué par une inflexion politique.

Dans sa première synthèse, explicitement dirigée aux Européens, Glauber énonce sa formule de l'«esthétique de la faim » (1965), lors de sa communication présentée à Gênes, dans le cadre de la Rétrospective du cinéma latino-américain organisée par l'Institut Columbianum. Plus tard, il assume le projet du "Cinéma Tricontinental ", inspiré de la Conférence Tricontinentale de La Havane (1967), avec un impact considérable dans le domaine du cinéma politique latino-américain. Ce projet est un motif récurrent dans le discours critique de Glauber, entre 1967 et 1970, mais son texte à plus large répercussion en cette période est "L'esthétique du rêve " (1971), exposé pour la première fois aux Etats-Unis. Cet article lui permet d'ajuster ses principes aux nouvelles options stylistiques présentes dans ses films postérieurs à Terre en transe ; son programme acquiert une forme nouvelle, reprenant des questions déjà présentes dans l' " esthétique de la faim ", mais rendant plus explicite sa mise à distance du réalisme et de ce qu'il désigne comme la raison bourgeoise.

C'est, par conséquent, dans un jeu politique d'une large ampleur qu'émergent ses textes, traduisant dès le début une articulation entre réflexion critique et création esthétique. En 1963, son livre Revisão crítica do cinema brasileiro ${ }^{14}$ apparaît comme un appel dirigé aux Brésiliens; en 1965, «Pour une esthétique de la faim », comme nous l'avons indiqué, est déjà un manifeste révélant le cinéaste conscient de son rôle dans un forum international : en s'adressant à des interlocuteurs européens, sa prémisse est celle de la confrontation inévitable, et son discours porte sur l'absence de compréhension, « de la part du développé », de l'expérience du Tiers Monde, soulignant ce qu'il perçoit comme une condition structurelle de l'altérité (dans le rapport entre l'Europe et l'Amérique latine). A partir de 1967, les interviews mettent en évidence l'ajustement stratégique entre le ton du propos, les concepts mobilisés et la définition de l'interlocuteur (type de revue, critiques avec lesquels un dialogue s'engage).

Révision critique a été écrit avant Deus e o diabo na terra do sol; "O Cinema Novo e aventura da criação $\|^{15}$, après Terre en transe. "L'esthétique du rêve " l'a été après $O$ dragão da maldade et ses premiers films à l'étranger, Der Leone et Têtes coupées. Dans cette interaction entre textes et films, il y a le combat qui s'engage à l'écran et celui qui s'engage dans les journaux et les revues. Le militantisme dans l'espace des médias projette la personnalité du cinéaste, donne de la résonance à ses mouvements, mais ouvre aussi la voie à la sédimentation de certains raccourcis vers une fausse interprétation de son cinéma.

En effet, destinés au combat, ses textes sont parfois excessifs, ce qu'il est possible d'expliquer comme un trait de personnalité, mais l'armature générale, appuyée sur des crédits incontestables, fait prévaloir le sens de la biographie comme l'expression d'une époque, document historique où le public et le privé sont inséparables, où une logique plus large (collective, historique) englobe et explique ce que, vu de près, pourrait apparaître comme l'expression d'une psychologie individuelle très proche de ce qu'il rejetait comme le théâtre bourgeois de l'expérience amoindrie. Glauber assume sa condition de personnage d'un théatre plus vaste, dans lequel il finit par incarner ce qui, par référence à l'héros désirable de ses films, constitue l' " homme brésilien multiple " (l'expression est de Glauber et elle reprend une formule de Mario de Andrade, écrivain et leader du modernisme dans les années 1920). Il importe ici de rappeler la réflexion autour des contradictions entre le poétique et le politique qui avaient traduit l'angoisse 
du jeune homme engagé, conscient de l'impossible expression radicale (et libre) de la subjectivité dans le cinéma. En tant qu'homme d'action, le cinéaste serait différent du poète, non seulement en raison de son rapport aux techniques et aux équipements, mais aussi parce que contraint par l'économie politique du cinéma dans une conjoncture défavorable.

Dans son sentiment afflictif de l'urgence - dont le corrélatif esthético-formel se retrouve dans le drame - tout converge. Et la discussion entreprise par Glauber des idées-force qui portent son cinéma ne se fait pas dans les termes classiques de l'idéologie, au sens strict de la construction d'un imaginaire qui légitime des intérêts ou même dans le sens général de "vision du monde » comprise en tant que formulation rationnelle d'une position dérivant de la connaissance, de la réflexion ou de valeurs proclamées. Le problème de la position et de l'intervention, chez Glauber, se trouve en deçà ou au-delà de cette sphère rationnelle, bien que sans l'exclure. La Raison, en tant que terrain exclusif de dispute de la vérité (ou de la légitimité d'une action), est un préjugé bourgeois, instrument de la répression et des manœuvres coloniales ethnocentriques, antipopulaires, menées au préjudice des trois continents qui composent la géopolitique de la faim. Ce n'est donc pas à partir de la réflexion des intellectuels enseignants que l'opprimé atteint la lumière et s'engage dans la résistance, mais par ce qu'il vit dans sa propre expérience, génératrice du sentiment d'absurde et de révolte, source de la violence qui fait l'histoire.

Il n'est pas surprenant que ce soit autour de la légitimation de cette violence - non pas "primitive ", mais en vérité civilisatrice - que le théâtre épique didactique de Glauber s'est fait à l'écran. Il convient d'ajouter, épique didactique dans ses propres termes, non pas dans ceux de Brecht, une inspiration incorporée et célébrée, mais sans grande articulation en termes de méthode et de présupposés. Chez Glauber, Bertold Brecht a cohabité avec Antonin Artaud, l'étrangeté critique avec la demande du théâtre comme peste, de l'art comme rituel agressif, viscéral, expérience de choc.

Depuis «Pour une esthétique de la faim » jusqu'à ses textes sur l'épique didactique écrits en 1975, en passant par "L'esthétique du rêve", le ton dominant a été l'affirmation de l'art comme laboratoire d'expérimentation des conflits à tous les niveaux : (1) formel - contre le cinéma classique et le réalisme ; (2) dramatique - contre l'approche psychologisante qui cultive l'autonomie de la sphère privée de l'expérience ; (3) thématique - l'impératif de mettre en scène toute expérience marquée par sa grande résonance sociale, se faisant au croisement des destins collectifs, théâtre du pouvoir qui mobilise de grands intérêts et des projets de mise en forme de l'histoire et de construction des identités nationales, continentales.

101 Flagrant chez Glauber, c'est son sens de la géopolitique (dont le cinéma est un des vecteurs), en tant qu'axe d'une confrontation et d'une étrangeté dans laquelle l'opprimé ne devient visible (et sujet éventuel dans ce processus) que par la violence. S'appuyant sur Frantz Fanon et sa célèbre analyse de la violence anticoloniale ${ }^{16}$, il explicite un tel sentiment dans « Pour une esthétique de la faim », en mettant l'accent sur la délimitation des lieux et le conflit structurel qui dérive de la barrière économique et sociale, culturelle et psychologique qui sépare l'univers de la faim du monde développé.

102 Frantz Fanon observe que lutter pour la culture nationale signifie avant tout lutter pour la libération nationale et construire une base matérielle rendant possible la formation d'une culture. Dans la situation historique qu'il évoque, il était 
incontournable de faire passer la lutte pour la culture par la voie de la guerre populaire.

Il y a une ressemblance et une différence entre le discours de Rocha, dans sa formulation de l' "esthétique de la faim ", et celui de Fanon, qui parle d'une lutte concrète de libération nationale, alors en cours dans les pays colonisés (Afrique, Asie), tandis que le cinéaste brésilien opère une transposition de niveau, en se référant à la production culturelle et non à une lutte armée. Le colonialisme que Rocha combat est le colonialisme culturel, et son idée de révolution brésilienne concerne un projet socialiste, non exactement un mouvement de résistance à l'occupation territoriale et à un ordre juridique dicté par des étrangers. Le recours au modèle de la «condition coloniale ", toujours fort dans les propositions de sortie du sous-développement dans le Brésil des années 1950-60, devient plus intense dans le champ du cinéma, où l'on connaît l'hégémonie exercée par les Etats-Unis. En ce sens, l' "esthétique de la faim » vise à la légitimation du cinema novo en tant que cinéma non aligné aux conventions de «l'autre»- l'industrie internationale - en refusant avec véhémence l'imitation de Hollywood.

Une fois reconnue la différence des situations, il faut pourtant souligner que Rocha et Fanon affirment un principe commun : la légitimité de la violence comme seule option du colonisé face à la domination subie. Dans les deux cas, opère la dialectique du maître et de l'esclave, dans les termes hégéliens : l'affirmation de l'opprimé se fait par la négation de l'oppresseur qui le nie. La formulation de Rocha révèle l'influence, dans le contexte brésilien d'alors, de Jean-Paul Sartre, penseur souvent cité par les idéologues de la révolution brésilienne qui élaboraient la question du " projet » et de l'affirmation nationale dans les termes de la phénoménologie du sujet chez Sartre.

Ce n'est pas par hasard que cette téléologie de la conscience opérant par négation et avançant vers la liberté joue un rôle structurant dans le Dieu noir, l'expérience créatrice majeure de Rocha à l'époque de son manifeste. Dans celui-ci, l'expression « esthétique de la faim » exprime non simplement les thèmes présents dans les films, mais surtout une façon de faire du cinéma. "De la faim ", donc, pour caractériser la pratique du cinéaste dans les conditions matérielles du sous-développement, pratique génératrice d'une nouvelle identité par la négation. Rocha vise à écarter un sens naïf de l'universel assumé par toute une tradition de la critique brésilienne et aussi par certains cinéastes; en même temps, et encore une fois en phase avec Fanon, il veut éviter la régression acritique et romantique aux valeurs d'un passé folklorisé. Sa posture est prospective et, s'il y a une défense du populaire, celle-ci se fait dans d'autres termes, comme il l'affirme dans "l'esthétique du rêve " (1971) et comme on le voit toujours clairement dans la forme et dans le style de ses films.

Il ne s'agit pas seulement d'une défense de la teneur sociale et politique des questions évoquées dans les films du cinema novo, mais d'avoir recours à l'espace sémantique de la faim pour se référer à la situation pratique des cinéastes, souligner leur effort d'invention formelle à partir de la rareté des ressources, dans une réponse plus lucide à la conjoncture historique qui produit des effets sur leur travail. C'est là le jalon décisif de l'invention d'une nouvelle esthétique laquelle, en raison d'un tel geste, atteint une portée universelle, non pas par l'effacement des particularités locales mais, au contraire, parce qu'elle fait de l'approche pertinente de ces particularités la condition nécessaire pour se faire reconnaître en dehors de tout paternalisme. La différence 
stylistique s'engendre dans la confrontation, elle a trait à une agression inévitable c'est une esthétique de la violence.

La vocation révolutionnaire de l'art s'affirme, pour Glauber, dans cette confrontation géopolitique qui implique des conflits ethniques, de classes et transnationaux. Cependant, si l'art est une pratique humaine insérée dans le champ symbolique, la défense de sa dimension politique d'affrontement et de risque n'élimine pas l'autre impératif : celui de comprendre que le plus grand impact du poétique sur le social dérive de sa nature même en tant qu'art. Comme il l'explique dans "L'esthétique du rêve ", l'art, car invention, c'est une plongée dans l'imprévu, expérience instauratrice, rupture avec le sens commun, avec les limites et les conventions. Il exprime la condition historique (et cosmique) dans sa totalité. En dehors du contrôle de la raison et de la mesure, il instaure ce qui n'est pas, assume le moment magique, entre en phase avec ce dont rêve l'opprimé, donne la parole à des pulsions inconscientes.

L'art, comme la révolution, c'est l'anti-raison. Il communique les tensions et rébellions face à l'insoutenable, exprimant ce qu'il y a d'imprévisible dans la pratique historique, la possession de l'homme qui projette sa vie en direction d'une idée. Si l'art est "surréel, expressionniste, délirant ", il ne l'est pas en tant qu'écriture automatique, comme le voulait le lyrisme particulier du mouvement surréaliste, mais comme rapprochement en profondeur (tel que seul l'art peut l'entreprendre) de l'imaginaire populaire (celui-là même qu'on désigne mysticisme). L'artiste doit incorporer cet imaginaire (le mystique), point nodal de la pauvreté, pour pouvoir produire les signes de la lutte corrélatifs de l'émotion stimulante et révélatrice. De tels signes extraits de la culture populaire apportent des condensations et, en cela, sont comme des idéogrammes (ici la référence vise Eisenstein). Voilà la matière dont se compose l'écriture du cinéaste engagé dans la libération de l'inconscient de la culture cristallisé en tant que mythe. Il ne s'agit donc pas de quelque chose qui serait susceptible d'être évaluée avec les paramètres du réalisme esthétique - tout particulièrement, avec les notions d'une critique inspirée de Georg Lukács voire d'Antonio Gramsci. Pour Glauber, il ne s'agit pas de reproduire l'humanisme de la Raison ni le programme du national populaire classique, à l'italienne, qui a acquis une grande place au sein de la critique brésilienne ${ }^{17}$.

Bref, le mot d'ordre réitéré ici est celui de la composition du cinéma épique didactique comme un rituel de l'anti-raison, contre le théâtre-cinéma en tant qu'institution bourgeoise, au profit d'un art en phase avec le mythe populaire dans une appropriation matérielle (de corps, geste et parole) libératrice face au consensus fabriqué par l'ordre social. Si la mystique, en tant que langage populaire de la rébellion, est un moment d'expression incompris par la raison, pour l'artiste, néanmoins, elle est un champ d'expérimentation tonifié par l'énergie révolutionnaire qui vient de l'inconscient (collectif) auquel le cinéaste a accès.

110 Une telle position de radar, d'interprète privilégié de la société, aurait trouvé dans le cinema novo une de ses modalités, non pas la seule, puisqu'il est précisé que l'art émancipateur peut avoir une dimension plus militante (le "Tiers Cinéma», de Fernando Solanas) ou une dimension radicale d'expérimentation et d'invention apparemment apolitique, comme celle cristallisée dans l'œuvre de Jorge Luis Borges. Ce dernier a « produit les irréalités libératrices de notre temps, son esthétique est celle du rêve ${ }^{18}$, dans la manifestation la plus abstraite, épurée, d'une tendance de la littérature latino-américaine à laquelle Glauber s'identifiait. Finalement, ce qu'on a désigné 
comme le "réalisme magique " gardait des points de contact avec son cinéma et avec la façon par laquelle il a prôné l'appropriation d'imaginaires (l'inconscient de la culture) en proposant une esthétique peu adaptée à ceux qui déchiffrent des personnages selon une clé psychologique et résument l'équation politique au bourgeois déchiré et au petit bourgeois radical.

Glauber a cherché à réunir ce qui se trouvait en deçà du réalisme (l'allégorie médiévale ou baroque) et au-delà (le modernisme, les avant-gardes). Si on tient compte de la triade classique - l'épique, le lyrique et le dramatique - son cinéma s'est voulu une synthèse particulière de ces trois approches. D'où son insistance sur l'épique didactique, compris dans sa connexion avec le mythe (à l'instar de la tragédie classique), avec la trame du pouvoir (à l'instar du drame baroque) et avec la quête d'une dimension épique qui comprend des transformations historiques de grande portée. Si l'horizon est la révolution, ce grand théâtre doit, d'un autre côté, laisser s'exprimer les pulsions inconscientes dans l'épaisseur poétique d'une lyrique moderniste avec laquelle Glauber a toujours dialogué. Du début jusqu'à la fin, ce qui se réaffirme c'est la tendance inclusive, totalisante, requérant de plus en plus la discontinuité et la juxtaposition pour réaliser son projet d'incorporation des forces vivantes de la culture.

112 L'esthétique du cinema novo s'est engendrée dans un cadre spécifique marqué par l'affirmation de cinématographies nationales et par le sens aigu du cinéphile engagé dans l'appropriation de ce qu'il assumait comme une vocation du milieu et de la technique à se connecter à l'expérience sociale, pour autant que l'image et le son se produisissent en dehors des limites de la simulation industrielle. On connait l'incorporation - dans le long métrage de fiction - d'un style de regard et d'écoute auparavant lié au documentaire, style générateur d'une expérience originale de contact sensible avec le monde, exclusive du cinéma, quelque chose de précieux qui ne se réduit pas au terrain de la représentation. Ce mouvement a lieu dans différents pays et il est mené par une génération qui, avec des motivations existentielles et politiques variées, a assumé son amour pour le cinéma et a formé sa sensibilité et son rapport au monde à partir de cette médiation spéciale.

113 Glauber est rentré sur scène dans ce contexte spécifique, non reproductible, marqué par l'affirmation du cinéma moderne de l'après-guerre, pariant sur la puissance de la mise en scène filmique en tant qu'expérience révélatrice. C'était le cinéma en tant que formation, découverte de l'histoire, vécu par une génération impatiente qui n'a pas voulu séparer l'art et la vie, la poésie et l'intervention dans le débat politique.

114 Il faut, par conséquent, envisager sérieusement une première implication du cinéma : l'appréhension sensible du monde par l'image et le son change complètement notre rapport aux «thèmes » de réflexion et à l'invention esthétique. Ce n'est pas un hasard si Glauber nous rappelle que le cinema novo a apporté ce qu'il appelle "sa contribution affective » à la connaissance du Brésil, "puisqu'il a discuté sur le vif de l'image et du son de ce qui auparavant n'était que statistique $»^{19}$. Cependant, ce mélange de cinéphilie et de préoccupation nationale, d'esthétique moderne et d'engagement politique, n'aurait eu la portée qu'il a pu avoir sans le sens pratique des jeunes face à un autre défi : celui de l'économie politique (comment rendre viable, comment inventer un cinéma dans un cadre de sous-développement économique ?). Là se trouve la deuxième implication du cinéma: puisque industrie, phénomène de masse, lutte de marchés, circulation de l'image et affirmation du pouvoir, il exprime un ensemble complexe de questions allant de l'art à la technique, de la culture à l'argent, et aussi les rivalités 
nationales, raison pour laquelle le cinéma est une condensation de capacité productive et valeur symbolique. Au XX ${ }^{\text {ème }}$ siècle, il a joué des rôles auparavant assumés par les foires industrielles, en tant que vitrine des conquêtes nationales sur une scène marquée par la compétition violente au nom du progrès; en vérité, une vitrine plus efficace, car affichant la présence routinière, quotidienne, des valeurs des pays centraux partout dans le monde, et non pas seulement au moment précis des grands événements. A la périphérie du système mondial est restée la pression d'une scandaleuse asymétrie géopolitique à laquelle le cinema novo a été si sensible et à laquelle il a cherché à apporter de nouvelles solutions.

En ce sens, il y a toujours eu cet « engagement sur tous les fronts » qui a fait tant de fois de l'intellectuel une figure du déchirement. Non par hasard, il s'agit là d'un thème explicite dans le cinéma de Glauber, notamment dans Terre en transe. Lorsque nous entendons la phrase prononcée par le personnage de Sara, «la politique et la poésie, c'est trop pour un seul homme ", une première réaction est de voir là-dedans avant tout un geste de consolation pour alléger les maux de Paulo Martins, le poète. D'ailleurs, le film même serait un exemple éloquent de négation de la phrase en question, lorsqu'il favorise une jonction notable de ces deux efforts, jonction qui, pour le reste, a défini toute la vie de Glauber Rocha. A l'occasion de sa mort précoce, certains ont trouvé dans la voix de Sara la résonance qu'ils recherchaient : elle aurait raison, et l'expérience du cinéaste écrivain politique, infatigable, invasif et vulnérable, aurait confirmé la dimension de sacrifice suggérée dans le propos protecteur auquel il n'a pas prêté l'oreille. Vraie ou fausse, cette inscription de l'expérience du cinéaste dans la clé d'une passion de l'histoire en tant que souffrance fait écho à ce que son cinéma a exprimé en tant que pensée sur l'histoire : apanage de la violence, en elle ne prennent leur essor que les interventions à caractère titanique. Il convient de dire alors que son terrain était celui de l'auto exigence sans trêves, active, exaspérée.

116 Toujours en tension avec la conjoncture, Glauber a été impatient. Tout dans son parcours mélange vie, œuvre et société. Ce qui ne veut pas dire que notre tâche à nous, pour le comprendre, soit limitée à l'évocation de la nature de son entreprise, renonçant à l'observation approfondie de son cinéma, marque de son charisme et de son leadership. En ce qui me concerne, j'admire entre autres la densité avec laquelle il a soutenu une permanence de style, une cohérence formelle apte à exprimer une dialectique de la fragmentation et de la totalisation qui marque, dans différents arrangements, l'ensemble de son cinéma. Si la dimension schématique était présente, le recours au mythe en tant que cadre de l'observation, il ne s'agissait pas là d'une impulsion dans le sens de congeler le temps dans des clés préalablement connues. Son interaction avec un monde changeant demandait des mouvements exploratoires, incertains, où le présent était assumé en guise d'ouverture.

117 L'ambivalence a été, depuis le début, la marque de son style. Dans ses films, les drames de l'histoire s'inscrivent dans la forme, comme c'est le propre du "cinéma de poésie » (pour employer la formule de Pasolini). Ici, la caméra se fait sentir, elle est d'une agilité remarquable, tantôt en conjonction tantôt en disjonction avec la mise en scène, créant des tensions entre des espaces ouverts et des délimitations théâtrales. Les personnages se condensent comme des emblèmes, mais sont observés par une caméra à l'épaule à la manière du documentaire, qui tâte des corps et des superficies. Le regard de Glauber est tactile, sensuel, même si le cadre de sa représentation est allégorique. La cohabitation des contraires est, en l'occurrence, typiquement baroque, dans la texture de l'image et 
du son, dans la conception du théâtre du pouvoir, ce que sanctionne l'évocation répétée du mot «baroque » lorsque Glauber se réfère à son propre cinéma. Ainsi soit-il. Mais à cette réserve près que tout cela s'est passé dans une conjoncture historique déterminée, et qu'il ne s'agit pas, par conséquent, de le comprendre en tant que manifestation d'un soi-disant « caractère national » incarné par le cinéaste.

Les contradictions productives de Glauber ont porté parce qu'il a fait son entrée sur scène à un moment spécifique marqué par l'affirmation du cinéma moderne de l'aprèsguerre, avec son nouveau regard et sa nouvelle écoute, avec son pari sur la puissance du film en tant que lieu de production d'une expérience inaugurale. Refusant les formules d'effet immédiat, il a créé des films plus complexes que ceux qu'exigeait la pédagogie militante. De cette façon, il a inventé un cinéma rare qui l'a projeté dans la constellation des interprètes de l'expérience continentale, un cinéma capable de jonctions inattendues, car tout se passe comme s'il s'était inspiré en même temps d'un Eduardo Galeano et d'un Octavio Paz. Autrement dit, comme s'il réunissait la volonté de faire l'anatomie de la spoliation d'un continent et la pensée sur sa destinée à partir d'un théâtre des mentalités sur le long terme. Sur ce dernier versant, culturaliste, où le symbolique a tendance à prendre son envol et s'autonomiser, Glauber, avec sa lucidité spéciale, a su se maintenir ancré dans des situations concrètes, en soumettant l'univers du mythe à la dure épreuve de la politique et de l'histoire.

\section{Bibliographie $^{20}$}

Glauber Rocha a commencé très jeune à publier des articles dans des périodiques brésiliens, d'abord à Bahia et, par la suite, dans différents organes nationaux. Ces textes ont d'abord donné lieu à deux livres, publiés alors qu'il était encore en vie. Revisão crítica do cinema brasileiro (Révision critique du cinéma brésilien), Rio de Janeiro, Ed. Civilização Brasileira, 1963, a été réédité en 2003 à São Paulo par Cosac \& Naify, avec une préface d'Ismail Xavier ; il a aussi donné lieu à une édition cubaine datée de 1965 (Ed. ICAIC, La Habana) et à une édition espagnole datée de 1971 (Ed. Fundamientos, Madrid). Revolução do Cinema Novo (Révolution du cinéma novo), 1981, Rio de Janeiro, Ed. Alhambra et Embrafilme, a été réédité en 2004, São Paulo, Cosac \& Naify, également avec une préface d'Ismail Xavier.

D'autres textes de Glauber Rocha ont donné lieu à des publications posthumes. Il en est ainsi de $O$ Século do cinema (Le siècle du cinéma), publié en 1985 à Rio de Janeiro par Alhambra et Embrafilmes, dont la sortie en France est prévue pour 2006, à Bobigny, chez Le Magic Cinéma ; et de Glauber Rocha, cartas ao mundo (Letres au monde), organisé par Ivana Bentes, et publié en 1977 à São Paulo par la Companhia das Letras.

En France, quatre études critiques sont particulièrement à signaler. En 1973, sous la direction de Michel Estève, paraît Le Cinéma Novo 2: Glauber Rocha, recueil de quatre articles publié dans Etudes cinématographiques 97/99, 111 pages. En 1974, René Gardies publie, dans la collection cinéma des éditions Seghers, Glauber Rocha (avec des textes et des propos du cinéaste). En 1987, Sylvie Pierre publie une biographie, Glauber Rocha, aux Eds. Cahiers du cinéma, Paris. Cette biographie, qui inclut des textes et des entretiens de Rocha, a fait l'objet d'une traduction brésilienne publiée à Campinas en 1996, chez Papirus. Enfin, en 2005, Le Magic Cinéma, en partenariat avec la Cinémathèque française, et dans le cadre de l'année du Brésil en France, a publié, sous la direction de Dominique Bax, Cyril Beghin et Mateus Araujo, Glauber Rocha. Anthologie du cinéma 
brésilien des années 60 aux années 80. Nelson Rodrigues, dans le cadre du tome 16 de la collection "Théâtres au cinéma ». Une bibliographie mise à jour et très complète des textes du cinéaste y figure. Sont encore particulièrement à signaler les deux études critiques de Lino Miccichè publiées en Italie : Glauber Rocha, saggi e inventtive sul nuovo cinema, 1986, Rome, éd. RAI et Glauber Rocha: scritti sul cinema, 198, Venise, éd. La Biennale, à l'occasion de la $43^{\text {ème }}$ Mostra internationale du cinéma.

\section{NOTES}

1. Ce texte est l'extrait d'une longue interview accordée, le 29 avril 1969, par Glauber Rocha à Fernando Cardenas et Rene Capriles, réalisée à Rio de Janeiro et publiée sous le titre « Glauber : el 'transe' da América Latina » dans Hablemos de cine, Lima (47) 34-38, mayo-jun. 1969. La traduction a été effectuée à partir de la version brésilienne qui figure dans Revolução do Cinema Novo /Glauber Rocha, São Paulo, Cosac \& Naify, 2004, recueil de textes du cinéaste publiés sous la coordination éditoriale d'Ismail Xavier et Augusto Massi, avec une préface d'Ismail Xavier.

2. $\mathrm{NdT}$ : Terre en transe évoque un pays imaginaire, Eldorado, où un coup d'état se prépare. C'est une allégorie de la situation brésilienne qui a précédé le coup d'état du 1 avril 1964 : des forces « réactionnaires » et des forces "progressistes » s'affrontent, mais il n'y a chez le cinéaste aucune empathie vis-à-vis de ces dernières, que le film décrit comme empêtrées dans la démagogie. Effectuant une critique puissante du populisme régnant dans les années 60 , le film était fait pour déplaire non seulement aux militaires mais aussi à une certaine gauche que beaucoup considéraient responsable de la prise du pouvoir par les forces armées.

3. NdT : Il s'agit de Nelson Pereira dos Santos.

4. $\mathrm{NdT}$ : Les Cahiers du cinéma, bien entendu.

5. «Uma estética da fome ». Revista Civilização Brasileira, Rio de Janeiro 1(3) : 165-170, jul. 1965. Reproduit in Revolução do Cinema Novo/Glauber Rocha, São Paulo Cosac \& Naify, 2004, pp. 63-67, avec la note explicative suivante : «Celle-ci est une des versions de l'exposé effectué par Glauber Rocha lors du séminaire 'Terzo Mondo e Comunità Mondiale', qui a eu lieu à l'occasion de la V Rassegna del Cinema Latino-Americano à l'initiative de la Comumbianum, à Gênes, 1965. D'autres versions existent avec différents titres, y compris celui d' "Esthétique de la violence", en français. »

6. $\mathrm{NdT}$ : Gouverneur de la ville-état de Rio de Janeiro, devenue état fédéré après le transfert de la capitale de la République vers Brasilia et alors dénommée "Etat de Guanabara ", entre 1960 et 1965. Ancien journaliste, puis homme politique, Carlos Lacerda a été à l'origine des scandales qui ont abouti au suicide du Président Getúlio Vargas en 1954.

7. NdT : 1961-1964. Janio Quadros, élu président de la République en janvier 1961, a donné sa démission pour des raisons obscures huit mois plus tard. Son successeur, le vice-président João Goulart, a été renversé par un coup d'état militaire le 1 avril 1964.

8. Esztetyka do sonho 71. Glauber Rocha, USA, Columbia University, 1971. Reproduit in Revolução do cinema novo/Glauber Rocha. São Paulo, Cosac \& Naify, 2004, pp. 248-251.

9. Ismail Xavier (Escola de Comunicações e Artes, Universidade de São Paulo).

10. Le « cinema novo » émerge au Brésil dans les années 1960 et s'inscrit dans un mouvement international de renouvellement du cinéma de l'après-guerre, à l'exemple de la «nouvelle vague » en France. 
11. Voir par exemple Revolução do Cinema Novo, São Paulo, 2004 - recueil de textes établi par Glauber Rocha avant sa mort et présenté dans sa forme éditoriale actuelle par Cosac \& Naify avec une préface d'Ismail Xavier; voir aussi $O$ século do cinema, Rio de Janeiro, 1985 - une nouvelle édition est actuellement préparée par Cosac \& Naify et doit paraître également en France, en 2006, chez Le Magic Cinéma.

12. Titre français : « Le dieu noir et le diable blond».

13. Titre français : « Antonio das mortes».

14. « Révision critique du cinéma brésilien ».

15. "Le cinema novo et l'aventure de la création ».

16. Voir Les damnés de la terre - Paris, Maspéro, 1961 pour l'édition française.

17. A l'occasion de la sortie de $O$ dragão da maldade contra o santo guerreiro aux Etats-Unis, Glauber s'est engagé dans une polémique où il a ébauché ses idées pour le texte "L'esthétique du rêve ". En réponse à la note critique de R. Callenbach, "Comparative Anatomy of Folk-Myth Film », in Film Quarterly Winter 1969-70, Glauber expose ses idées anti-réalistes et son interprétation du lien entre mythe et inconscient, refusant la manière par laquelle l'auteur fait appel à Gramsci, Lukács et Freud pour opposer des restrictions à son film. Voir l'article "The Way tou Make a Future » (dialogue entre Glauber et Gordon Hitchens), in Film Quarterly Fall 1970.

18. Voir "L'esthétique du rêve », dans ce dossier.

19. Voir Revolução do Cinema Novo, 2ème édition revue et améliorée, São Paulo, Cosac Naify, 2004, p. 115.

20. Pour d'autres informations sur le cinéaste et son oeuvre on peut consulter le site : http:// www.tempoglauber.com.br

\section{AUTEURS}

\section{ANGELINA PERALVA}

Angelina Peralva est sociologue, franco-brésilienne, professeure à l'université de Toulouse II, chercheure au CADIS-CNRS/EHESS, Paris) et associée au CIRUS (CNRS-Université de Toulouse II). Ses recherches portent sur les liens entre violence et démocratie, avec des terrains en France et au Brésil.

\section{ISMAIL XAVIER}

Ismail Xavier est polytechnicien, licencié de l'Ecole de Communication et Arts deSão Paulo, docteur en théorie littéraire et professuer de l'ECA-USP. 